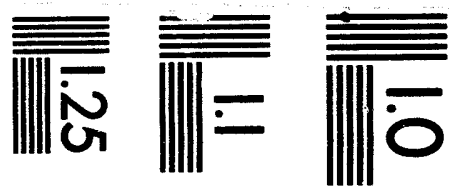

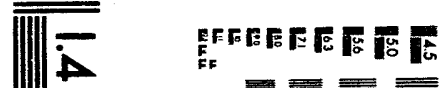

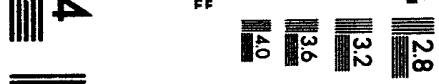

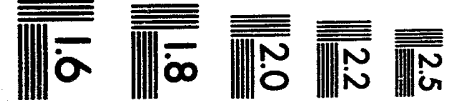



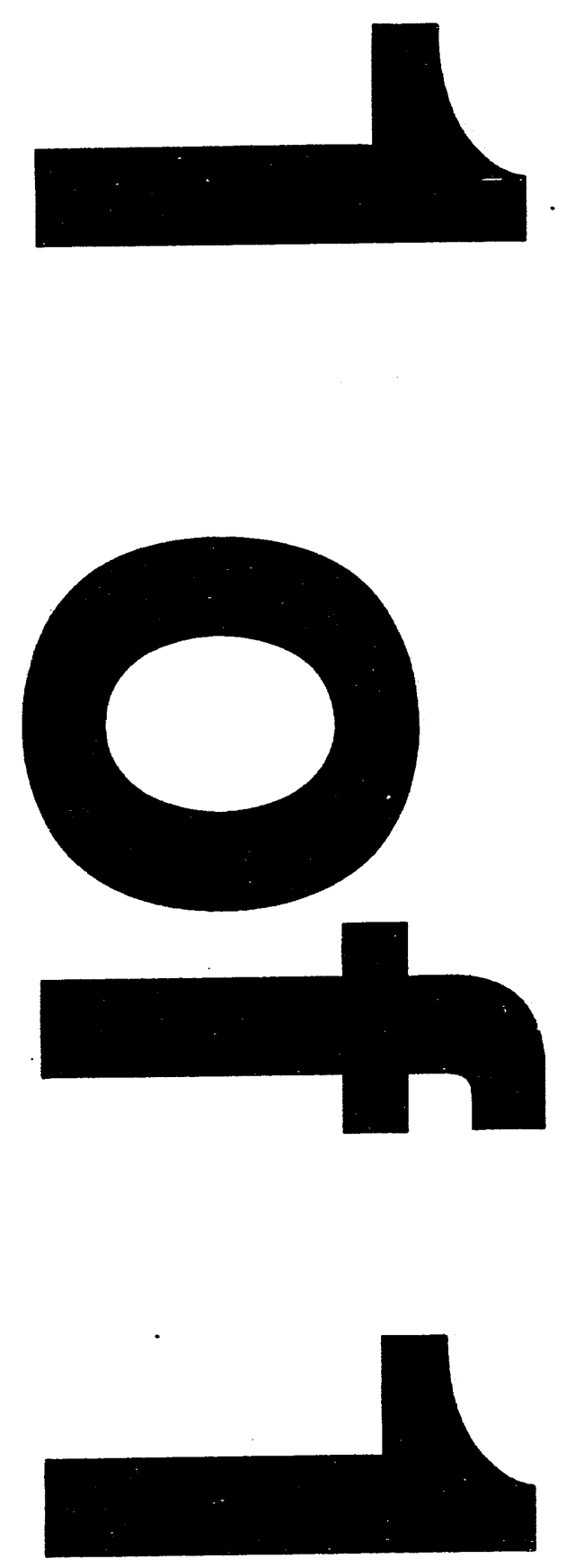


\title{
Linac Coherent Light Source (LCLS) at 2-4 nm using the SLAC Linac*
}

\author{
J. T. Seeman, K. Bane, R. Boyce, G. Loew, P. Morton, H.-D. Nuhn, J. Paterson, P. Pianetta, \\ T. Raubenheimer, R. Tatchyn, V. Vylet, H. Winick \\ Stanford Linear Accelerator Center, Stanford University, Stanford, CA 94309 \\ C. Pellegrini, J. Rosenzweig, G. Travish \\ Department of Physics, University of California (UCLA), Los Angeles, CA 90024 \\ D. Prosnitz, E. T. Scharlemann \\ Lawrence Livermore National Laboratory, Livermore, CA 94550 \\ K. Halbach, K.-J. Kim, M. Xie \\ Lawrence Berkeley Laboratory, University of California, Berkeley, CA 94720
}

\begin{abstract}
We describe the possible use of the SLAC linac to drive a unique, powerful, short wavelength Linac Coherent Light Source (LCLS) ${ }^{1,2}$. Using the FEL principle, lasing is achieved in a single pass of a high peak current electron beam through a long undulator by self-amplified-spontaneous-emission (SASE). The main components are a high-brightness electron RF gun with a photocathode, two electron bunch length compressors, the existing SLAC linac, beam diagnostics, and a long undulator combined with a FODO quadrupole focusing system. The RF gun, to be installed about $1 \mathrm{~km}$ from the end of the SLAC linac, would produce a single bunch of $6 \times 10^{9}$ electrons with an invariant emittance of about $3 \mathrm{~mm}$-mrad and a bunch length of about $500 \mu \mathrm{m}$. That bunch is then accelerated to $100 \mathrm{MeV}$ and compressed to a length of about $200 \mu \mathrm{m}$. The main SLAC linac accelerates the bunch to $2 \mathrm{GeV}$ were a second bunch compressor reduces the length to $30-40 \mu \mathrm{m}$ and produces a peak current of $2-3 \mathrm{kA}$. The bunch is then accelerated to $7-8 \mathrm{GeV}$ and transported to a $50-70 \mathrm{~m}$ long undulator. Using electrons below $8 \mathrm{GeV}$, the undulator could operate at wavelengths down to $2 \mathrm{~nm}$, producing about $10 \mathrm{GW}$ peak power in sub-ps light pulses. At a linac repetition rate of $120 \mathrm{~Hz}$, the average power is about $1 \mathrm{~W}$. Linac operation at lower beam energies provides longer wavelength radiation. After the undulator, the beam is deposited in a dump. The LCLS light pulses are then distributed to multiple user stations using grazing incident mirrors. Length compression, emittance control, phase stability, FEL design criteria, and parameter tolerances are discussed. A demonstration experiment is also described which uses the SLAC linac and (possibly) the PALADIN undulator to study SASE to power saturation at wavelengths of 40-360 nm.
\end{abstract}

\section{INTRODUCTION}

Two recent developments have given hope to the possibility of constructing linac-based $x$-ray lasers operating at short wavelengths, down to $2 \mathrm{~nm}$ and eventually down to $0.1 \mathrm{~nm}$. First, at Los Alamos (and elsewhere) RF photocathode guns have been shown to deliver low emittance $(3-4 \mathrm{~mm}$-mrad normalized), high charge $(>1 \mathrm{nC})$ electron beams. Second, the successful operation of the Stanford Linear Collider $(\mathrm{SLC})^{3}$ has demonstrated the required tools, understanding, and techniques needed to transport, accelerate, and compress electron bunches without dilution of phase space. These developments make it possible to deliver the electron beams with phase space densities sufficient to drive short wavelength lasers.

* Work supported by DOE offices of Basic Energy Sciences and High Energy and Nuclear Physics.

Presented at the SPIE 1993 International Symposium on Optics, Imaging, and Instrumentation, (Electron-Beam Sources of High Radiation Conference), San Diego, CA, July 11-16, 1993. 
This LCLS R\&D facility is aimed at the development of linac-driven, short wavelength $x$-ray lasers, and their scientific and technological utilization. The parameters of the LCLS are listed in Table 1 and an overview is shown in Fig. 1. After commissioning, the first laser would operate starting at a wavelength of about $10 \mathrm{~nm}$ and then be pushed to the $2-3 \mathrm{~nm}$ region. With more extensive $R \& D$, along with the use of higher energy electrons, additional undulators, and the development of improved RF guns, it is expected that this facility could achieve (with additional funding) a laser operating at even shorter wavelengths, possibly in the $0.1 \mathrm{~nm}$ regime.

Many facilities at SLAC make it an attractive site for the LCLS. The $50 \mathrm{GeV}$ linac is operational. Many beam diagnostics are installed and functional. An enclosure to house the undulator exists at the end of the SLAC linac. This area is the Final Focus Test Beam (FFTB) which was completed in 1993 for R\&D associated with final focus systems for future linear colliders. There is sufficient space in the FFTB housing to accommodate the LCLS undulator. After a slight upgrade, the FFTB enclosure would provide adequate shielding for beams delivered alternately to both facilities.

Table 1 Performance parameters of the $2-4 \mathrm{~nm}$ FEL using the SLAC Linac

\begin{tabular}{|l|l|}
\hline \multicolumn{1}{|c|}{ Parameter } & \multicolumn{1}{c|}{ Value for the $2-4 \mathrm{~nm}$ FEL } \\
\hline Electron energy & $7 \mathrm{GeV}$ \\
\hline Bunch charge & $1 \mathrm{nC}$ or $6 \times 10^{9}$ \\
\hline Electron beam emittance (normalized) & $3.5 \mathrm{~mm}-\mathrm{mrad}$ \\
\hline Bunch length (1 sigma) & $30-40 \mu \mathrm{m}$ \\
\hline Peak current & $>2000 \mathrm{~A}$ \\
\hline Peak coherent power & $>10 \mathrm{GW}$ \\
\hline Average coherent power & $1 \mathrm{~W}$ \\
\hline Pulse width & $<160 \mathrm{fs}$ \\
\hline Coherent photons / pulse & $>10^{14}$ \\
\hline Coherent energy / pulse & $3 \mathrm{~mJ}$ \\
\hline Repetition rate & $120 \mathrm{~Hz}$ \\
\hline Bandwidth (1 sigma) & $0.1-0.2 \%$ \\
\hline Peak brightness & $>10^{31}$ photons $/ \mathrm{s} / \mathrm{mm}^{2} / \mathrm{mrad}^{2}$ within $0.1 \% \mathrm{BW}$ \\
\hline Average brightness & $>10^{21}$ photons $/ \mathrm{s} / \mathrm{mm}^{2} / \mathrm{mrad}^{2}$ within $0.1 \% \mathrm{BW}$ \\
\hline Photon beam diameter at $50 \mathrm{~m}$ & $2 \mathrm{~mm}$ \\
\hline Beam divergence & $10^{-5} \mathrm{radians}$ \\
\hline
\end{tabular}

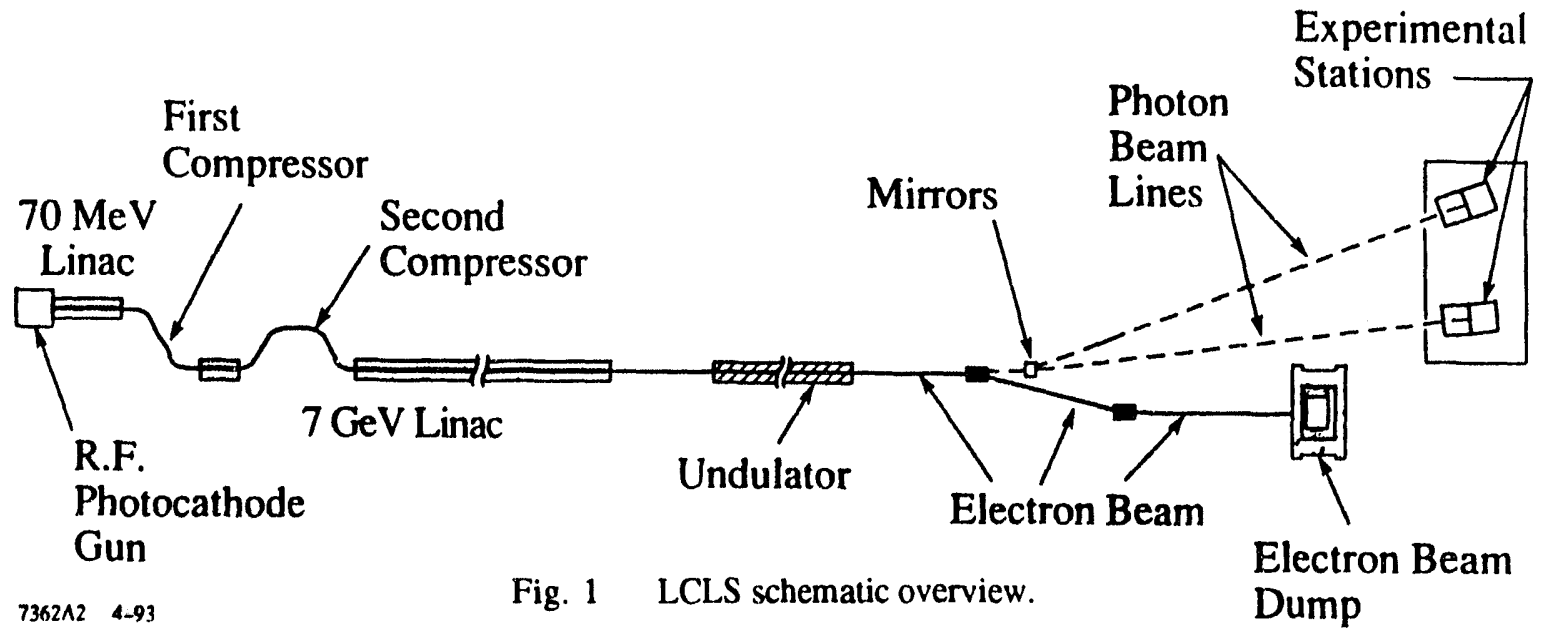


The LCLS photon beams emerge in: the SLAC research yard, about $125 \mathrm{~m}$ from the SSRL beam lines on SPEAR. Thus, it is possible to bring beams from the ICLS and SPEAR to the same sample chamber for pump-probe experiments.

The proposed LCLS operates on the principle of the FEL, but does not need an optical cavity which is difficult or impossible to make at such short wavelcngths. Instead, $x$-ray laser operation is achieved by self-amplified-spontaneousemission (SASE) in a single pass of an electron beam through the 50-70 m long undulator. Although SASE theory is well developed, there is little experimental data with which to compare, since most FELs have used oscillator cavities. It is therefore important to make detailed comparisons between experiment and theory, for example to verify the accuracy and wavelength dependence of simulation codes and assumptions about startup from noise. We plan to do this initially at wavelengths around $10 \mathrm{~nm}$ or longer, where certain tolerances are more manageable. As experience is gained and tighter tolerances are met, operation down to $3 \mathrm{~nm}$ can be expected, still using electrons below the $10 \mathrm{GeV}$.

From Table 1 the average values of the brightness and coherent power are about 3 orders of magnitude greater than the that projected for 3rd generation light sources such as the ALS and APS. Peak values are about 9 orders of magnitude higher. Photon beams with this extraordinarily high brightness, coherence, and peak power will make possible a wide range of experimental studies in many scientific and technical fields including $x$-ray imaging of biological specimens in and around the "water window" (including producing $x$-ray holograms of live biological specimens in a single sub-picosecond pulse), time resolved studies of condensed matter systems and chemical reaction dynamics, and non-linear processes. Because the propertics of this light source go many orders of magnitude beyond that available from any other source in operation or construction, it is likely that entirely new scientific applications will be opened. Exploratory experiments will be carried out on two diagnostic / experimental stations. With two experiments able to receive pulses, techniques will be developed for rapid switching of the beam, as well as rapid changing of the beam parameters such as wavelength and intensity to meet different experimental conditions. An FY 1996 Short Form Construction Project Data Sheet has been submitted to DOE for this project. The total estimated cost is $\$ 29.45 \mathrm{M}$.

\section{RF GUN}

We have studied the design of an RF photocathode gun ${ }^{4}$ which can produce the beam characteristics required for reliable operation of the LCLS. The dynamics of the photo-electron beam have been modeled using both PARMELLA and an axisymmetric particle-in-cell code (ITACA). These simulations show that a one $\mathrm{nC}$ bunch in a $31 / 2$ cell $2.856 \mathrm{GHz}$ structure has a pulse length of $2 \mathrm{psec}$, an rms energy spread of $0.2 \%$, and a normalized rms emittance of $3 \mathrm{~mm}$-mrad, as can be seen from simulations in Figs. 2 and 3. The major challenge in designing this source concerns reproducibility of the beam properties. In particular, due to wake-fields in the transport system it is critical that the jitter in the total charge per pulse and the injection timing must be minimized ${ }^{5}$. We believe that the solution to these problems exists, based on the choice of a rugged cathode material and a commercial diode-pumped laser system with timing stabilization ${ }^{4}$.

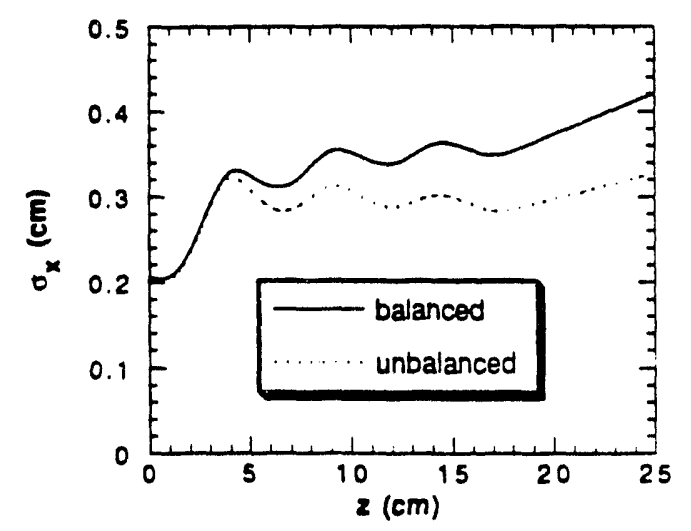

Fig. 2 Transverse beam envelope vs distance in the gun.

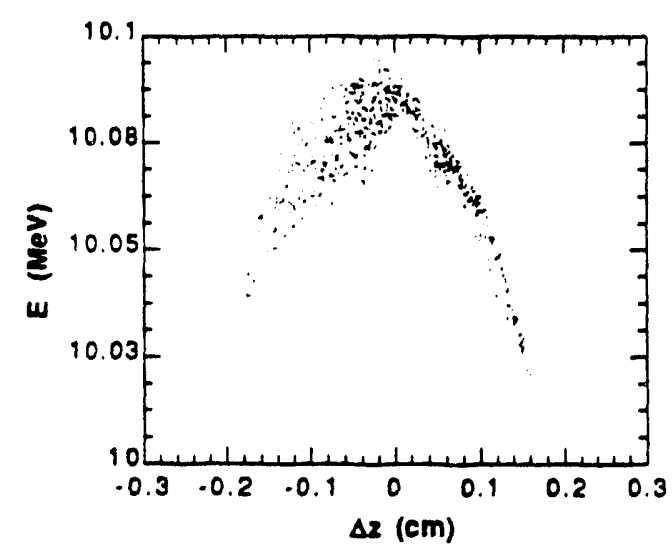

Fig. 3 Energy vs longitudinal position of $10 \mathrm{MeV}$ particles. 


\section{ELECTRON TRANSPORT}

The bunch produced by the LCLS photo-injector must be accelerated and length compressed before injection into the undulator. In the present scheme the bunch is accelerated from $10 \mathrm{MeV}$ to about $7 \mathrm{GeV}$ using three linear accelerators separated by two compressors 5 . A schematic view of the second compressor is shown in Fig. 4 . The final bunch length is about $40 \mu \mathrm{m}$ which is over a factor of 10 smaller than that produced by the photocathode gun yielding a peak current of 2500 to $3500 \mathrm{~A}$. The final energy spread is less that the required 0.1 to $0.2 \% \mathrm{~ms}$.

The choices of energies at which to compress are influenced by the need 1) to control the longitudinal wakefields for energy spread minimization, 2) to minimize emittance growth from transverse forces, and 3) to reduce the effects of timephase jitter as well as beam intensity jitter from the injector and in the compression process.

The emittance dilution effects due to transverse wakefield, RF deflections, and dispersive effects have been modeled in the SLAC linac for this configuration assuming $150 \mu \mathrm{m}$ random misalignments of the quadrupoles and beam position monitors, $300 \mu \mathrm{m} r \mathrm{~ms}$ random misalignments of the accelerating structures, and a transverse-longitudinal coupling of $\mathrm{g}_{\mathrm{rms}}=2 \times 10^{-4}$ for the RF deflections ${ }^{6}$. A transverse beam jitter equal to the rms beam size was also assumed. The computer simulation results which indicate the optimum bunch length after the first compressor are shown in Fig. 5. At a bunch length of $200 \mu \mathrm{m}$ (rms), we find a modest $25 \%$ emittance growth along the linac with an acceptable energy spread. Emittance growth after the second compression is negligible due to the short bunch length and small energy spread.

Longitudinally, the first compression is performed at $100 \mathrm{MeV}$ where the bunch length is reduced from $0.5 \mathrm{~mm}$ to 0.2 $\mathrm{mm}(\mathrm{ms})$. The second compression is near $2 \mathrm{GeV}$ and reduces the length to about $0.04 \mathrm{~mm}$. To study the development of longitudinal phase space in this process, a computer program was used considering the effects of longitudinal wakefields, curvature of the RF wave, and phase and intensity jitter. The second compression is made to deliberately over-compress the bunch length beyond the $25-30 \mu \mathrm{m}(\mathrm{rms})$ minimum. This slight over-compression and subsequent acceleration from 2 to 7 $\mathrm{GeV}$ allow appropriate cancellation of upstream errors, thus providing significantly relaxed timing and intensity jitter tolerances of the injector and accelerator RF. After the first compression the bunch length is still nearly gaussian, but after the second compression the beam distribution is more sharply spiked and has long tails. The final beam phase space, bunch length, and energy distribution are shown in Fig. 6. Note that between the horns of the bunch the peak current is everywhere $>5 \mathrm{kA}$ and that the full-width of the energy distribution is $0.04 \%$, both of which satisfy our requirements ${ }^{5}$. If energy collimation is done to remove the long energy tails, the peak current in the center of the bunch is about $3 \mathrm{kA}$. If we define the jitter tolerance as the amount of change in a parameter that changes the full-width of the bunch by $10 \%$, we find that in this example the tolerance to incoming current jitter is $2.2 \%$ and to incoming phase jitter is $0.45^{\circ}$. We believe these tolerances are achievable with recently available commercial equipment. (A second set of parameters for this length compression scheme has been studied which provides a longitudinal distribution which is more gaussian shaped. Both distributions produce the peak current and energy spread needed to satisfy the FEL requirements.) An experimental test of the second bunch compression including longitudinal and transverse effects has been designed and is under consideration?

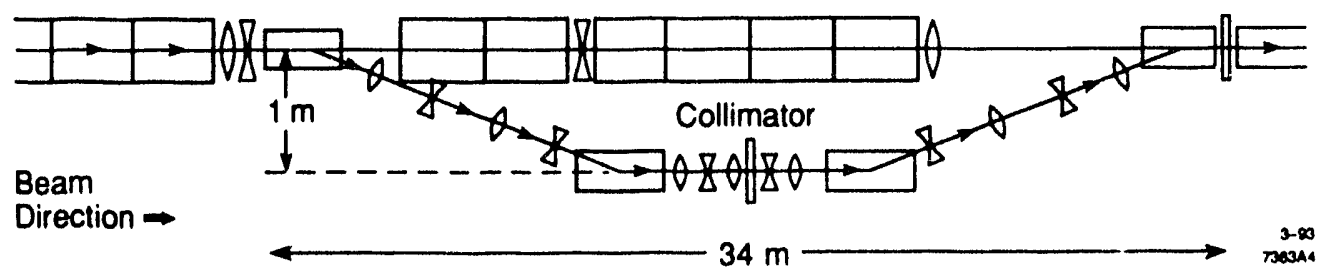

$\mathrm{Fi}_{\circ} .4$ Schematic overview of the second bunch length compressor. 


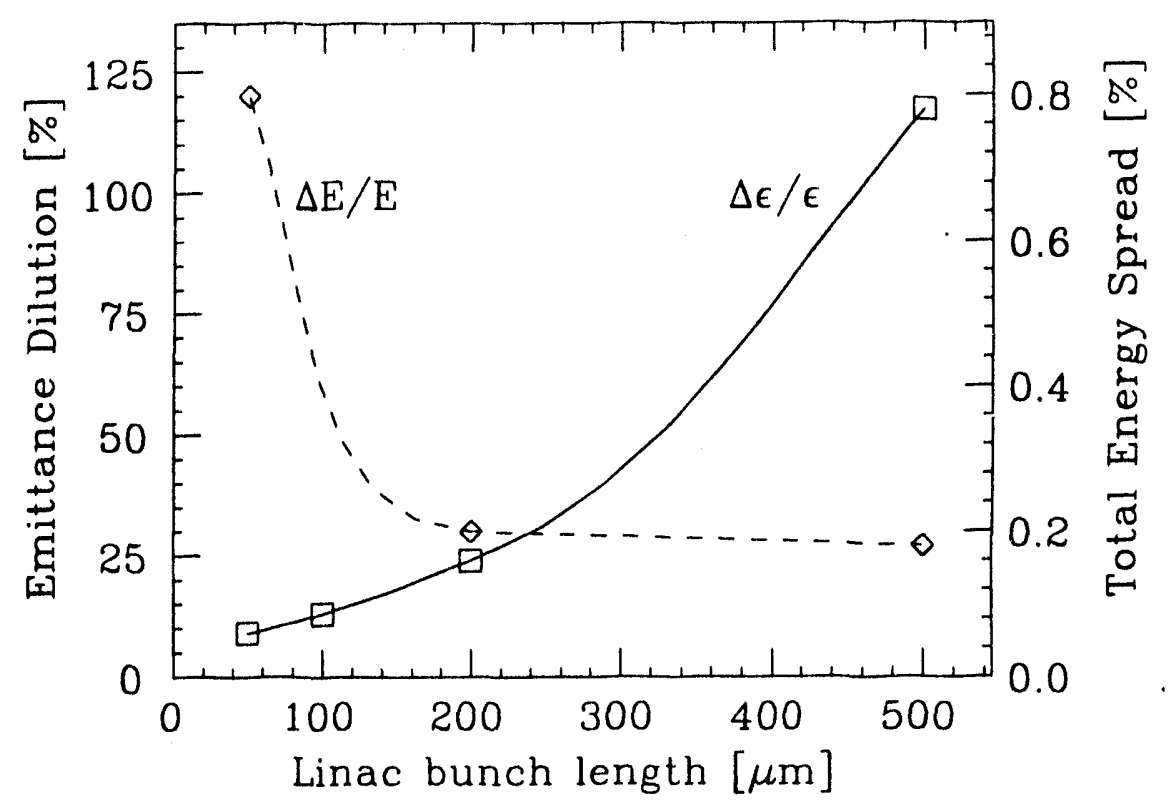

Fig. 5 Transverse emittance dilution (solid) and peak-to-peak energy spread (dashed) as a function of linac bunch length after the first compressor. We conclude that a bunch compressed to $\sigma_{\mathrm{Z}}=200 \mu \mathrm{m}$ at $100 \mathrm{MeV}$ and then compressed a second time at $2 \mathrm{GeV}$ to $40 \mu \mathrm{m}$ is optimal to provide the necessary peak current of over $2000 \mathrm{~A}$, to preserve the transverse emittance, and to maximize the allowed jitter tolerances.

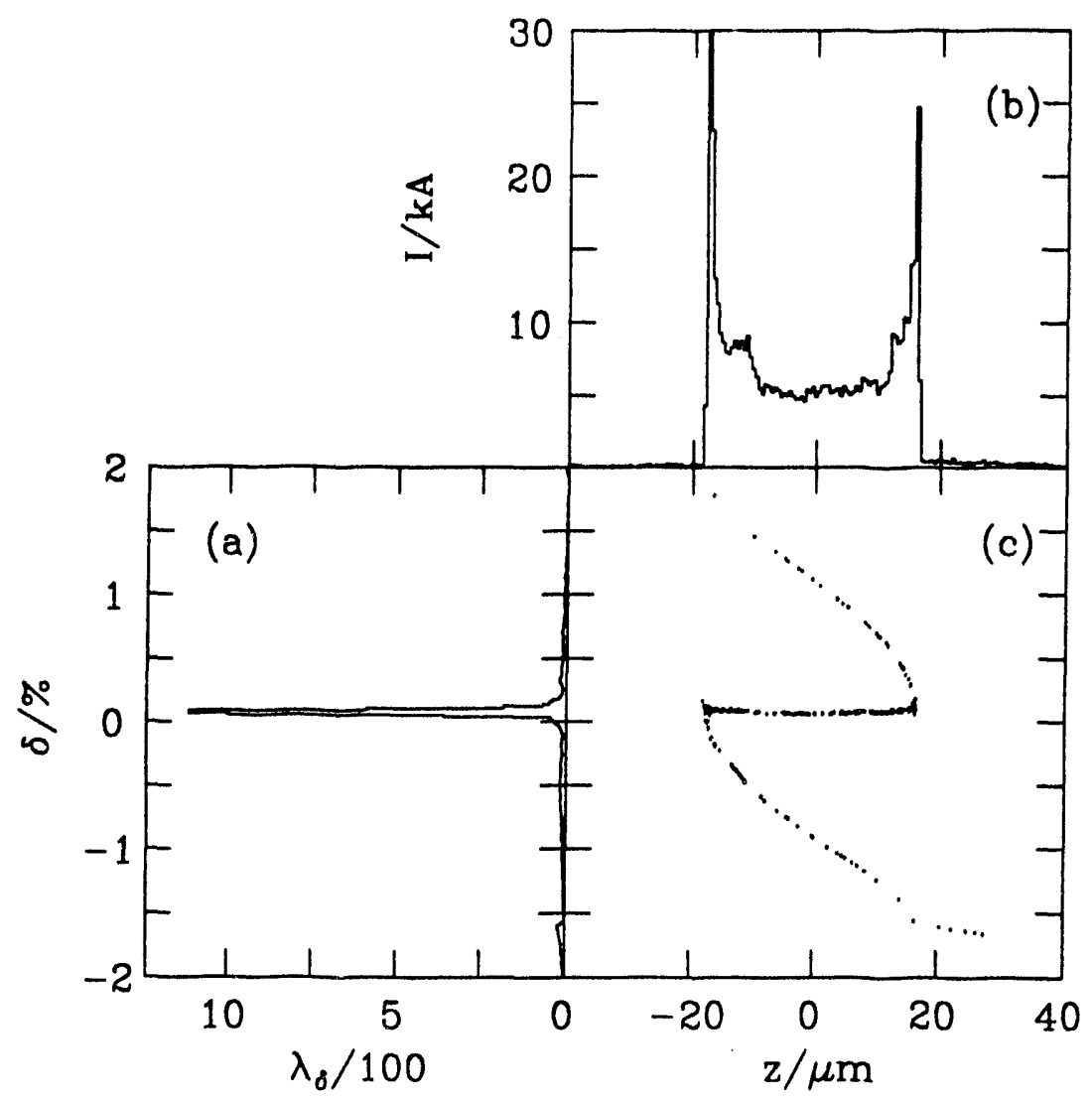

Fig. 6 The energy spread $\delta$ (a), the charge distribution (b), and the shape of phase space (c) at the end of the $7 \mathrm{GeV}$ linac. 


\section{LCLS PERMANENT MAGNET UNDULATOR}

The undulator design is based on permanent magnet (PM) material because of the broad base of experience acquired by the scientific community, continued improvement of commercially available PM materials, and the advantage of relatively straightforward analytical investigations. Extensive numerical simulations have been performed using the FRED3D and TDA3D codes, primarily, to obtain working design parameters for the required long undulator ${ }^{8}$. In agreement with simple models, the simulations predicted that the LCLS can provide in excess of $10 \mathrm{GW}$ of peak power in a sub-picosecond pulse. The saturation length is about $60 \mathrm{~m}$ with strong focusing provided throughout the undulator. The system gain, its optimization, and tolerances to beam parameters changes, wiggler errors and misalignments have been studied ${ }^{9}$.

Based on 3D simulations of a continuous single pass field structure, the following parameter set has been established for the LCLS undulator: a) period $=8 \mathrm{~cm}, \mathrm{~b}$ ) peak fields amplitude $=0.8 \mathrm{~T}, \mathrm{c}) \mathrm{K}=6, \mathrm{~d}$ ) total length $=60 \mathrm{~m}$, and e) focusing betatron wavelength $=60 \mathrm{~m}$. The pure permanent magnet undulator structure has a superimposed focusing (FODO) quadrupole lattice generated by $40 \mathrm{~cm}$ long $15 \mathrm{~T} / \mathrm{m}$ quadrupoles placed at $80 \mathrm{~cm}$ intervals. A schematic overview of the undulator is shown in Fig. 7 and the permanent magnet arrangement is shown in Fig. 8. Other numerical details are given in Table 2. With the possibility of tuning the 1st harmonic of the undulator by varying the beam energy, the conventional use of movable jaws was determined to be dispensable, making possible a design of relative simplicity. The PM material, spacer block., keeper, vacuum system, and supports are an integrated fixed arrangement. To facilitate orbit and phase correction, beam position monitors are spaced at $1.6 \mathrm{~m}$ intervals, with corrector dipoles located every $3.2 \mathrm{~m}$. Work on a short prototype section is in progress to help resolve engineering details, magnet tolerances, and fields measurement issues.

The operational parameters chosen provide relative insensitivity to beam current and emittance fluctuations. By running to saturation, variations in the output radiation due to changes in the beam parameters are minimized. Several results from simulations ${ }^{9}$ are shown in Fig. 9 indicating that a $60 \mathrm{~m}$ length is consistent with the electron beam properties expected from the injector and accelerator. The requirements on the uncorrelated energy spread of the beam are tight $(<0.04 \% \mathrm{rms})$ and are determined primarily by the desire to maintain a narrow bandwidth and maximum gain. Energy spreads twice as large as specified do not seriously degrade the (single frequency) performance. This, along with the high power (brightness) of the optical pulse, suggests that filtering could be used to narrow the line width.

According to the simulations using a random walk, the necessary wiggler field errors are small $(<0.2 \%$ rms field errors and $<5 \mathrm{mr}$ easy axis orientation errors) but are within present industry practices. Procedures to sort and match PM blocks are under development. Steering and alignment requirements are also tight $(30 \mu \mathrm{m} r \mathrm{~ms})$, yet less stringent than required for many future linear colliders designs.

Table 2 Parameters of the LCLS undulator and FODO quadrupole lattice.

\begin{tabular}{|l|l|}
\hline Permanent magnet lattice parameters & FODO quadrupole lattice parameters \\
\hline$\lambda \mathrm{u}=8 \mathrm{~cm}$ & Quad aperture radius $=6 \mathrm{~cm}$ \\
\hline$\lambda \mathrm{mu}=2 \mathrm{~cm}$ & Quad outside diameter $=20 \mathrm{~cm}$ \\
\hline $\mathrm{h}=1.9 \mathrm{~cm}$ & Quad length $=40 \mathrm{~cm}$ \\
\hline $\mathrm{w}=4 \mathrm{~cm}$ & Quad gradient $=15 \mathrm{~T} / \mathrm{m}$ \\
\hline $\mathrm{g}=1.5 \mathrm{~cm}$ & FODO period $=1.6 \mathrm{~m}$ \\
\hline $\mathrm{t}=1.9 \mathrm{~cm}$ & Phase advance per cell $=11.5$ degrees \\
\hline $\mathrm{Br}=1.08 \mathrm{~T}$ & Total power budget $=300 \mathrm{~kW}$ \\
\hline
\end{tabular}




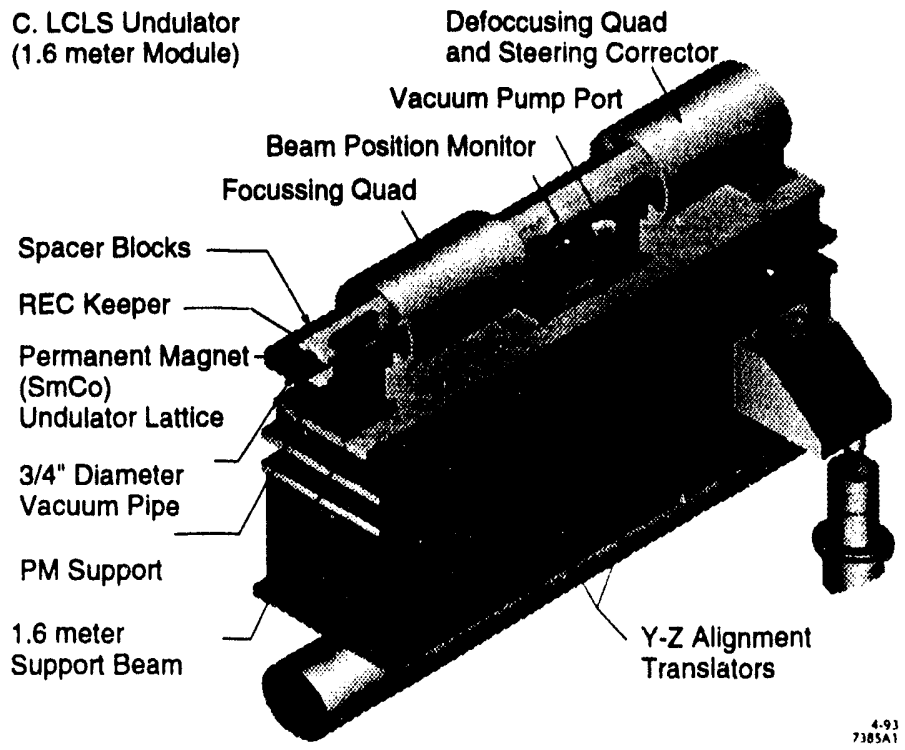

Fig. 7 Girder and component layout of the LCLS undulator.
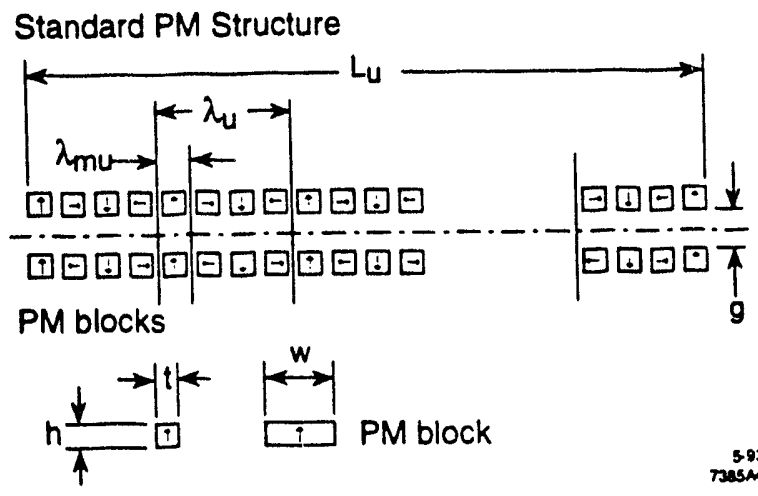

Fig. 8 "Split" Halbach permanent magnet configuration of the LCLS.
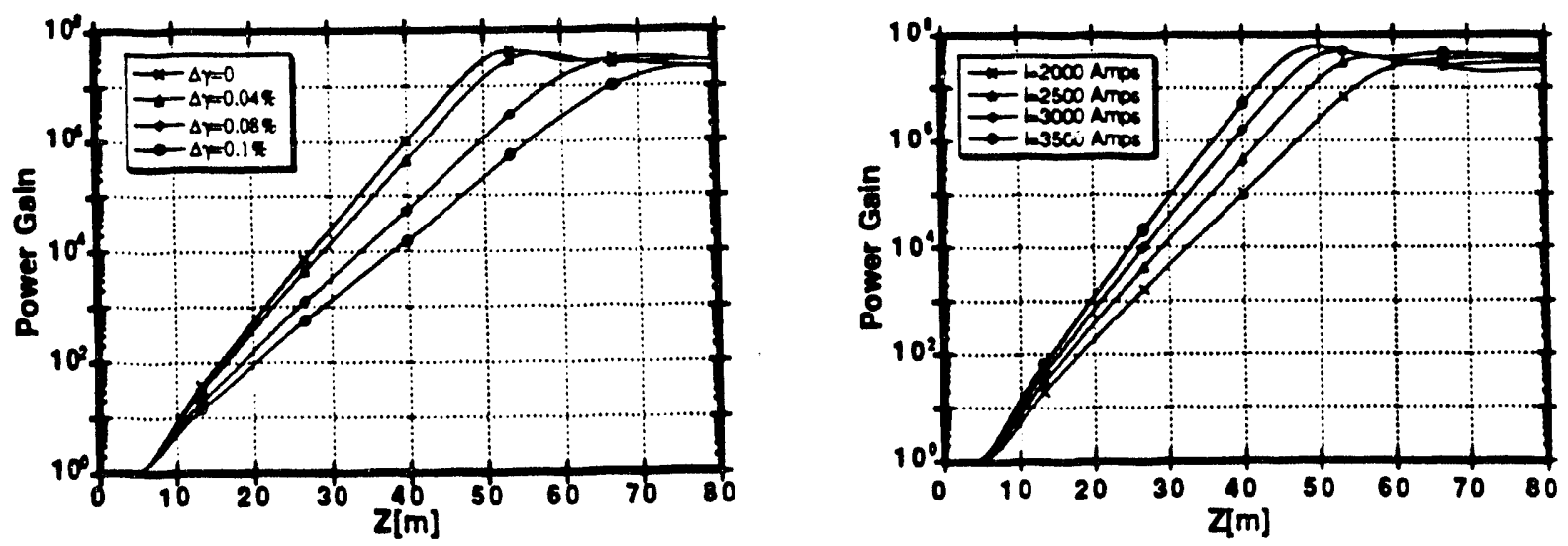

Fig. 9 Power gain curves for various uncorrelated energy spreads and peak beam currents. 


\section{PHOTON LINES AND USER STATIONS}

Due to the extreme brevity and peak intensity of the LCLS output radiation, special emphasis has been placed on the design of the photon beam line system. To minimize the likelihood of sustaining component damage at the expected $10^{12}$ $\mathrm{W} / \mathrm{cm}^{2}$ peak power densities at normal incidence, a deflection scheme utilizing solid-state mirrors at grazing incidence bas been developed ${ }^{10}$. An initial concept of this mirror system can be seen in Fig. 10. Furthermore, the necessity of maintaining high reflectivity to avoid peak-power damage leads to the need for an ultra-high vacuum environment with the provisions for in-situ cleaning of all the reflecting surfaces. To exploit the diffraction-limited source size of the LCLS, the use of a simple monochromator configuration utilizing a single grating in a conical diffraction geometry, with the source as the effective entrance slit, is under consideration. User facilities including beam lines, experimental areas, and beam shutters have an initial design. An overview is shown in Fig. 11.

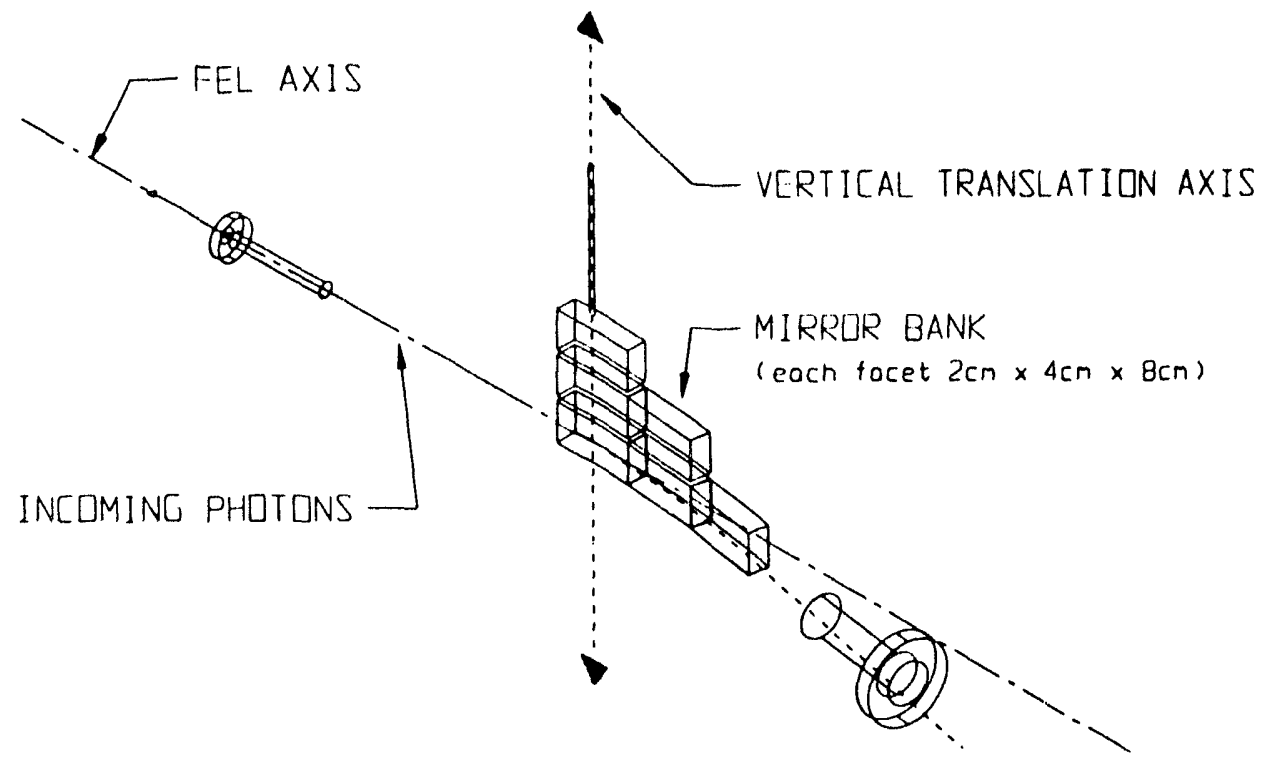

Fig. 10 Photon beam deflection mirrors, each having a 4 degree bend.

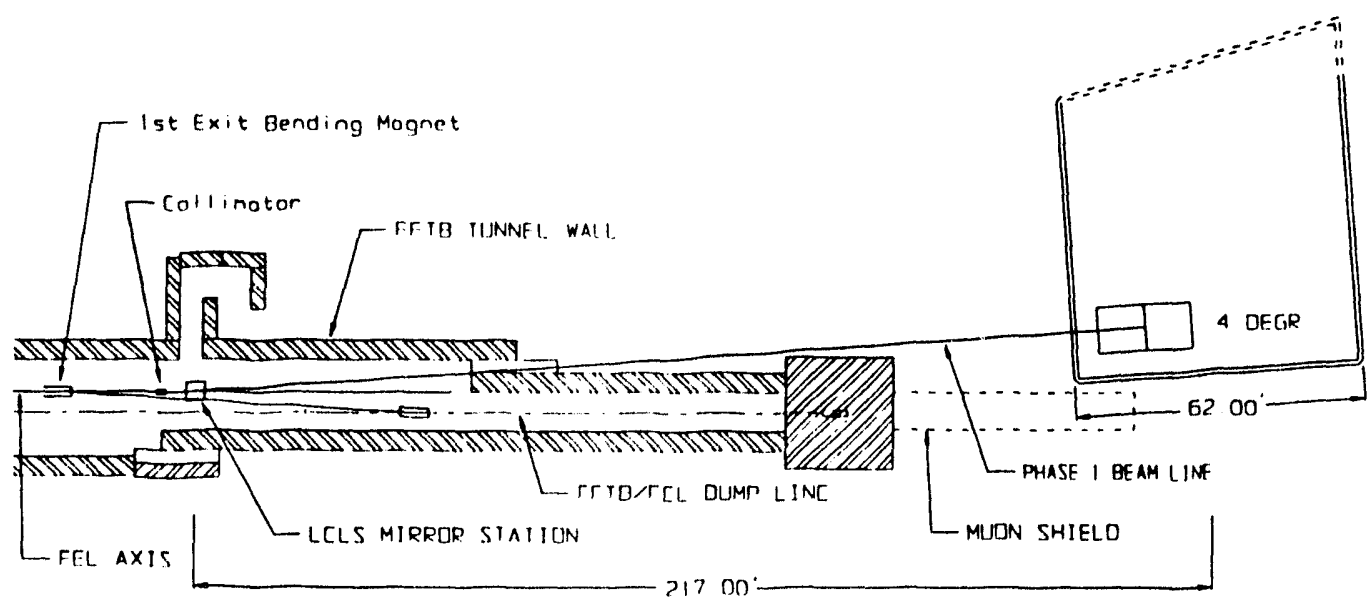

Fig. 11 Layout of the first photon beam line and user station relative to the undulator. 


\section{DEMONSTRATION EXPERIMENT}

A short wavelength FEL demonstration experiment using the SLAC Linac is being considered to test the beam manipulation and SASE lasing from startup in an undulator. This demonstration is aimed at an inexpensive FEL test in the 40-360 nm range at SLAC. The present idea would use no dedicated accelerator time during operation, but would have sufficient beam time obtained by parasitically obtaining a "few hertz" beams from other programs with minimal interference. The need for additional non-available hardware is minimized. The components would be installed in a few brief downtimes. The PALADIN undulator from LLNL will likely be made available for this application. The ultimate wavelength reach of this FEL experiment [ $\sim 40 \mathrm{~nm}$ ] [or lower without saturation] should be sufficiently short to go beyond the reach of other potential FEL experiments.

The studies made possible by this demonstration would be: 1) electron beam dynamics of double bunch compression and acceleration, 2) SASE laser startup as a function of electron beam charge, density, energy, and energy spread, 3) SASE fluctuations at startup, and 4) power saturation effects including tolerances and steering.

This FEL demonstration experiment would be located early in the SLAC linac (Sectors 1 and 2) where the ordinary beam energy is low. The experiment would be parasitic to the straight ahead "End Station A" runs which are available about 3 months per year. Thus, the linac quadrupole lattice need not be changed from the primary beam program. The beam would be injected into the FEL at about $1 \mathrm{~Hz}$ (or more) using DC and pulsed magnets to inject and extract the beam from the linac.

A schematic view of this full scale demonstration experiment is shown in Figure 12. The beam is generated in an RF photocathode gun, which is driven by "load" power from existing klystrons and accelerating structures in the first $100 \mathrm{~m}$ of the SLAC linac (Sector 1). A single bunch of $1 \mathrm{nC}$ is made in the gun, accelerated to about $70-100 \mathrm{MeV}$, and injected into the Sector 1 accelerator using the last bend magnet in the "SLC positron injection chicane" in early Sector 1. The first bunch length compression is done in this injection process. After acceleration to about $300-500 \mathrm{MeV}$, a pulsed chicane at the end of Sector 1 compresses the bunch length a second time to the required $30-40 \mu \mathrm{m}$. The emittance of the beam (about $3.5 \mathrm{~mm}$ mrad) is measured in early Sector 2 with existing profile monitor hardware. The beam is then accelerated to the final energy 500-1500 MeV in Sector 2 and extracted with an existing "pulsed dumper" magnet at Linac Girder 2-9. Finally, the beam is directed through a short transport line to the PALADIN undulator located in the aisle of the accelerator tunnel. PALADIN would be mounted parallel to the linac and displaced by about $0.75 \mathrm{~m}$. After passing through PALADIN, the spent electron beam is dumped. The emerging photon beam is bent vertically into a $10 \mathrm{~m}$ shaft and analyzed upstairs in the klystron gallery. The estimated parameters for this full scale demonstration experiment are in Table 3 with increased focusing in PALADIN. Power saturation can be achieved in all cases with field gain lengths of 1 to $2.2 \mathrm{~m}$. The demonstration would begin with natural focusing in PALADIN but with enhanced fields made by closing the undulator gap. Later, stronger focusing with permanent magnets inserted into the gap providing additional quadrupole terms will lower the electron betatron functions and shorten the field gain lengths by about $30 \%$.

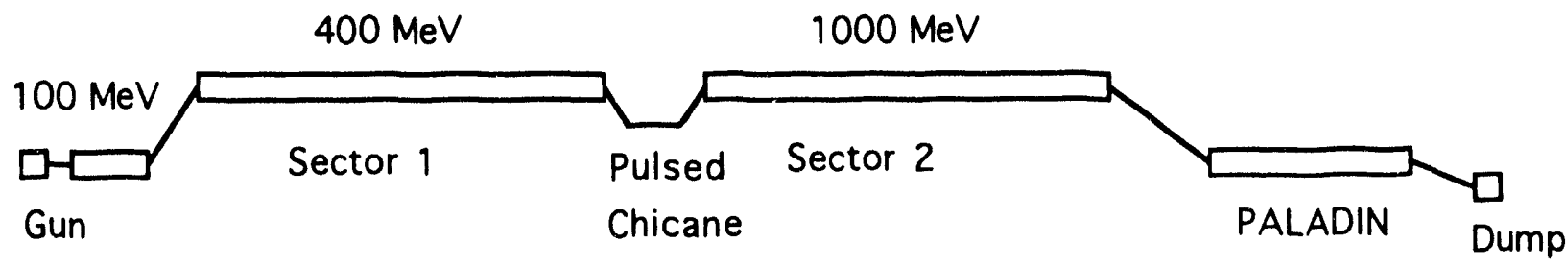

Fig. 12 Schematic layout of a possible demonstration SLAC-FEL at 40-360 nm using PALADIN. 
Table 3 Parameters of a SLAC demonstration FEL using PALADIN with extra focusing $(\beta=2 \mathrm{~m})$ and stronger fields.

\begin{tabular}{|l|l|l|l|}
\hline Parameter & Low Energy & Medium Energy & High Energy \\
\hline $\mathrm{e}^{-}$energy (GeV) & 0.51 & 1.02 & 1.53 \\
\hline$\gamma$ & 1000 & 2000 & 3000 \\
\hline $\mathrm{e}^{-}$energy spread (\%) & 0.02 & 0.02 & 0.02 \\
\hline$\gamma \varepsilon(\mathrm{x}, \mathrm{y})$ mm-mrad & 3.5 & 3.5 & 3.5 \\
\hline Ipeak $(\mathrm{A})$ & 2000 & 2000 & 2000 \\
\hline Bunch length $(\mu \mathrm{m})$ & 40 & 40 & 40 \\
\hline Undulator period (m) & 0.08 & 0.08 & 0.08 \\
\hline Undulator K & 4 & 4 & 4 \\
\hline Undulator field (kG) & 5.5 & 5.5 & 5.5 \\
\hline$\lambda$ (nm) & 360 & 90 & 40 \\
\hline Field gain length (m) & 1.05 & 1.6 & 2.2 \\
\hline Saturation length (m) & 11 & 18 & 24 \\
\hline Power saturation $(\mathrm{GW})$ & 4.4 & 5.7 & 6.0 \\
\hline
\end{tabular}

\section{Z. ACKNOWLEDGMENTS}

This project benefited greatly from a technical review in November, 1992. We thank the committee members: 1 . Ben-Zvi

(Chairman), J. Bisognano, L. Elias, J. Goldstein, B. Newnam, K. Robinson, R. Schlueter, A. Sessler, and R. Sheffield.

\section{REFERENCES}

1. C. Pellegrini, et al, "A 2 to $4 \mathrm{~nm}$ High Power FEL on the SLAC Linac," International FEL Conference, Kobe, Japan, August 24-28, 1992, and NIM A331, p. 223-227, 1993.

"2. H. Winick, et al, "A $2-4 \mathrm{~nm}$ Linac Coherent Light Source (LCLS) Using the SLAC Linac," Proceedings of the IEEE 1993 Particle Accelerator Conference, Washington, D.C., May 17-20, 1993.

3. J. Seeman, "Accelerator Physics of the Stanford Linear Collider and SLC Accelerator Experiments Towards the Next Linear Collider," Advances of Accelerator Physics and Technologies, H. Schopper, Ed., World Scientific, p. 219, 1993.

4. J. Rosenzweig and L. Serafini, "Design of a High Brightness RF Photoinjector for the SLAC Linac Coherent Light Source," Proceedings of the IEEE 1993 Particle Accelerator Conference, Washington, D.C., May 17-20, 1993.

5. K. Bane, T. Raubenheimer, and J. Seeman, "Electron Transport of a Linac Coherent Light Source (LCLS) using the SLAC Linac," Proceedings of the IEEE 1993 Particle Accelerator Conference, Washington, D.C., May 17-20, 1993.

6. J. Seeman, et al., "RF Beam Deflections Measurements and Corrections in the SLC Linac," Proceedings of the IEEE 1985 Particle Accelerator Conference, Vancouver, B.C., Canada, p. 2629, May 13-16, 1985.

7. R. Holtzapple and J. Seeman, "Short Bunch Length and Low Emittance Test Area using the SLAC Linac," Proceedings of the IEEE 1993 Particle Accelerator Conference, Washington, D.C., May 17-20, 1993.

8. R. Tatchyn, R. Boyce, K. Halbach, H.-D. Nuhn, J. Seeman, H. Winick, and C. Pellegrini, "Design Considerations for a 60 Meter Pure Permanent Magnet Undulator for the SLAC Linac Coherent Light Source (LCLS)," Proceedings of the IEEE 1993 Particle Accelerator Conference, Washington, D.C., May 17-20, 1993.

9. K.-J. Kim, M. Xie, E. T. Scharlemann, C. Pellegrini, and G. Travish, "Performance Characteristics, Optimization, and Error Tolerances of a $4 \mathrm{~nm}$ FEL Based on the SLAC Linac," Proceedings of the IEEE 1993 Particle Accelerator Conference, Washington, D.C., May 17-20, 1993.

10. R. Tatchyn and P. Pianetta, "X-Ray Beam Lines and Beam Line Components for the SL.AC Linac Coherent Light Source (LCLS)," Proceedings of the IEEE 1993 Particle Accelerator Conference, Washington, D.C., May 17-20, 1993. 

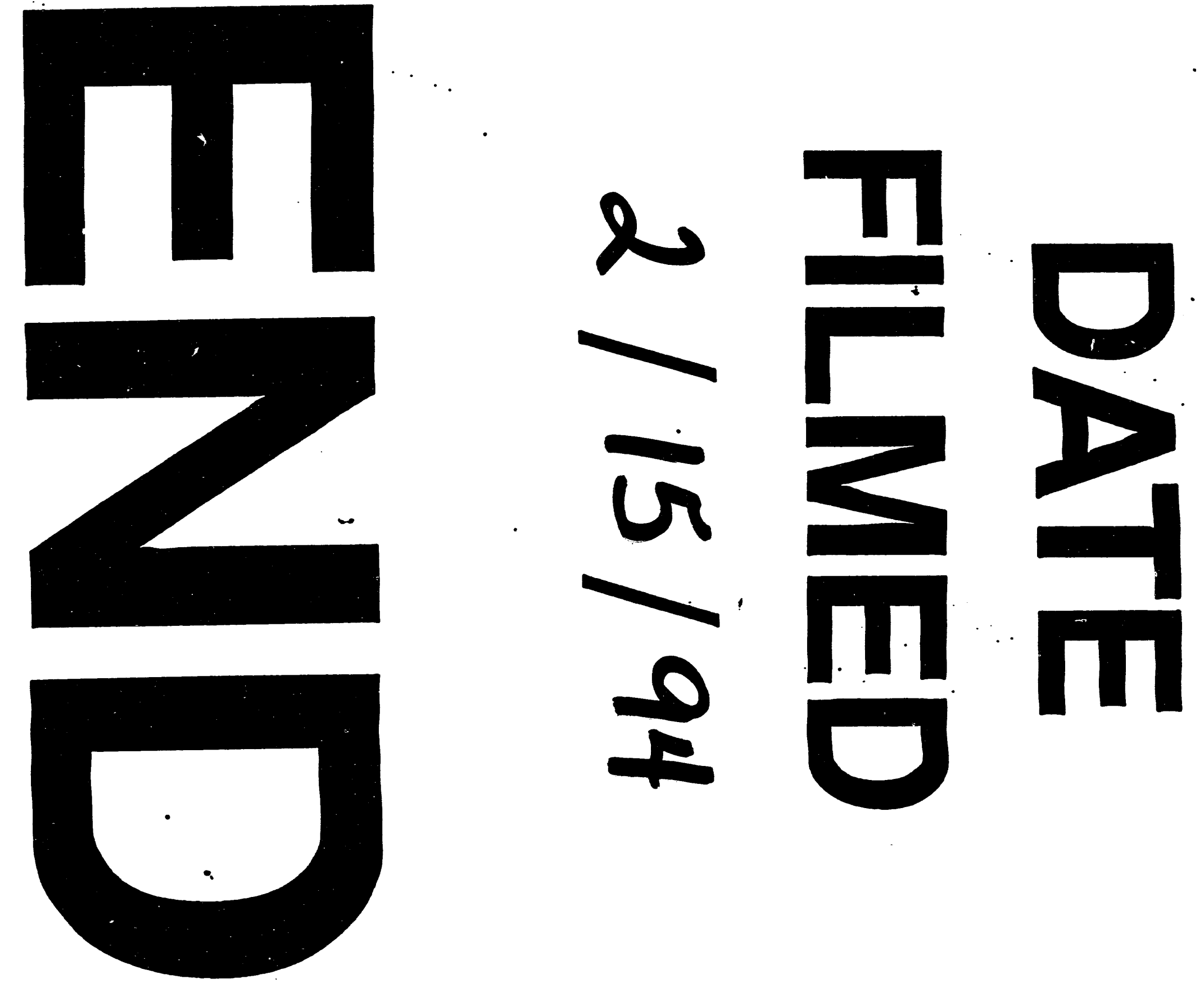